\title{
Technische Universität Graz
}

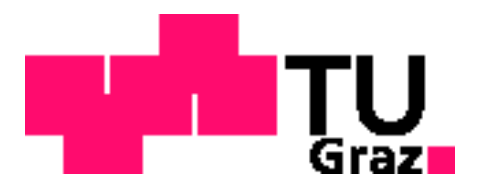

\section{On the Numerical Solution of Minimal Energy Problems}

G. Of, W. L. Wendland, N. Zorii

\section{Berichte aus dem Institut für Numerische Mathematik}





\section{Technische Universität Graz}

\section{On the Numerical Solution of Minimal Energy Problems}

G. Of, W. L. Wendland, N. Zorii

\section{Berichte aus dem Institut für Numerische Mathematik}


Technische Universität Graz

Institut für Numerische Mathematik

Steyrergasse 30

A $8010 \mathrm{Graz}$

WWW: http://Www.numerik.math.tu-graz.at

(C) Alle Rechte vorbehalten. Nachdruck nur mit Genehmigung des Autors. 


\title{
On the Numerical Solution of Minimal Energy Problems
}

\author{
G. Of* W. L. Wendland’ and N. Zorii \\ *Institut für Numerische Mathematik, Technische Universität Graz, \\ Steyrergasse 30/III, A 8010 Graz, Austria, \\ ${ }^{\dagger}$ Institut für Angewandte Analysis und Numerische Simulation, \\ Universität Stuttgart, Pfaffenwaldring 57, D 70569 Stuttgart, Germany, \\ ${ }^{\ddagger}$ Institute of Mathematics, National Academy of Science of Ukraine, \\ 3 Tereshchenkivska Str., 01601, Kyiv-4, Ukraine
}

\begin{abstract}
We study the constructive and numerical solution of minimizing the energy for the Gauss variational problem involving the Newtonian potential. As a special case, we also treat the corresponding condenser problem. These problems are considered for two two-dimensional compact, disjoint Lipschitz manifolds $\Gamma_{j} \subset \mathbb{R}^{3}, j=1,2$, charged with measures of opposite sign. Since this minimizing problem over an affine cone of Borel measures with finite Newtonian energy can also be formulated as the minimum problem over an affine cone of surface distributions belonging to the Sobolev-Slobodetski space $H^{-\frac{1}{2}}(\Gamma), \Gamma:=\Gamma_{1} \cup \Gamma_{2}$, which allows the application of simple layer boundary integral operators on $\Gamma$, a penalty approximation for the Gauss variational problem can be used. The numerical approximation is based on a Galerkin-Bubnov discretization with piecewise constant boundary elements. To the discretized problem, the projection-iteration is applied where the matrix times vector operations are executed with the fast multipole method. For the condenser problem, we solve the dual problem which reduces in our case to solving two linear boundary integral equations. Here the fast multipole method provides an efficient solution algorithm. We finally present some convergence studies and error estimates.
\end{abstract}

Keywords: Minimal Newtonian energy problem, dual condenser problem, Lipschitz surface, penalty method, simple layer boundary integral operator, boundary element approximation.

Mathematics subject classification (2000): 31C15 - 31C20 - 45A05 - 49J35 - 45L10 · $65 \mathrm{R} 20$

*email: of@TUGraz.at, Phone: +43 3168738123,FAX: +43 3168738621

†email: wendland@mathematik.uni-stutggart.de, Phone: +49 71168565235

†email: natalia.zorii@gmail.com 


\section{Introduction}

Carl Friedrich Gauss investigated in [5] the variational problem of minimizing the energy of the Newtonian potential over non negative charges on the boundary surface of a given domain. The sign condition was given up in connection with boundary integral equation methods where distributional boundary charges have been introduced for solving boundary value problems. (See e.g. Costabel's article [4] and also [9].)

A different generalization of the original Gauss variational problem maintaining the sign restriction but employing measures as charges has independently grown into an eminent branch of modern potential theory (see e.g. [14] and the extensive works [20][26] and for two dimensions [15]). In this paper, we consider this so-called generalized Gauss variational problem (from now on just Gauss problem) on two two-dimensional non intersecting compact Lipschitz surfaces $\Gamma_{1}, \Gamma_{2} \subset \mathbb{R}^{3}$ which are loaded by measures $\mu=\mu^{1}-\mu^{2}, \mu^{j} \in \mathcal{M}^{+}\left(\Gamma_{j}\right), j=1,2$, and try to determine the corresponding equilibrium state also numerically by appropriate boundary element approximation. To this end, we first show that every Borel measure with finite Newtonian energy defines uniquely a distribution $\sigma$ on $\Gamma=\Gamma_{1} \cup \Gamma_{2}$ belonging to the Sobolev-Sobodetski space $H^{-\frac{1}{2}}(\Gamma)$ such that the linear functional defined by the measure and the one defined by the $L_{2}$-duality with $\sigma$ coincide on $C^{\infty}(\Gamma)$. Moreover, the minimizing Gauss problem over the measures is equivalent to minimizing the energy over a corresponding affine cone in $H^{-\frac{1}{2}}(\Gamma)$ which can be expressed in terms of the simple layer boundary integral operator on $\Gamma$. This allows to approximate the Gauss problem by employing a penalty formulation. The latter corresponds to a nonlinear variational problem on the whole space $H^{-\frac{1}{2}}(\Gamma)$ whose solutions provide error estimates depending on the penalizing parameter.

For the penalized variational problem, the use of piecewise constant boundary elements on triangulations on $\Gamma$ and corresponding Galerkin-Bubnov discretization results in a convex, finite-dimensional minimization problem with a symmetric, positive definite system matrix. The latter can be solved by the gradient-projection method applied to the discrete system (see [6, Chapt. I]). For the corresponding matrix times vector multiplications, the fast multipole method [13] is used. The convergence of the gradient-projection method depends on the penalty parameter as well as on the boundary element's mesh-width and becomes very slow for large penalty and small mesh-width. In particular, the charges converge faster for smaller penalty whereas the total charge values are better approximated for larger penalty. Therefore we are using a cascading approach.

In [25], the dual problem to the Gauss problem is defined and it is shown that its solution coincides with the one to the original Gauss problem if the latter exists. If the exterior source of energy is zero then the Gauss problem becomes a condenser problem for which the dual formulation is easier to treat (see $[24,26]$ ). If the condenser consists of just two plates without irregular points as considered here, and for the special weight function $g=1$, the dual problem simplifies significally since then the potential values of the minimizing solution are constant on $\Gamma_{j}, j=1,2$ and sum up to 1 . In this case, the dual problem can be reduced to the solution of two linear boundary integral equations whose solution provides the desired equilibrium state. The latter can be approximated also by 
piecewise constant charges, and the fast multipole boundary element method provides here an efficient solution algorithm.

In applications, the numerical solution of the Gauss as well as of the condenser problem is of great interest if for practical reasons in electrical engineering on $\Gamma_{1}$ only nonnegative and on $\Gamma_{2}$ only nonpositive charges are allowed (see "capacitors" in [10]). The numerical solution of the condenser problem also has applications in approximation theory and the development of efficient numerical integration (see [7]).

We present numerical experiments for the Gauss as well as for the condenser problem. In particular, we show that the cascading approach for the penalty method can be used for the Gauss variational problem.

For the condenser problem, two types of examples a) and b) are presented where for $\Gamma_{2}$ surfaces are used which approximate an infinitely long surface. The corresponding numerical results are in agreement with theoretical results in [20, 23], where under the geometry of example b) it was shown that for the infinitely long surface an equilibrium minimizing measure does not exist, in the contrary to example a).

\section{The generalized Gauss variational problem}

We are going to consider the Gauss problem in $\mathbb{R}^{3}$, i. e., the problem of minimizing the Newtonian energy of signed Borel measures in the presence of an external field $f$. The corresponding class of admissible measures (or charges) is supposed to be associated with a condenser, which is treated here as an ordered pair $\mathcal{A}=\left(\Gamma_{1}, \Gamma_{2}\right)$ of two-dimensional compact, Lipschitz manifolds $\Gamma_{1}, \Gamma_{2} \subset \mathbb{R}^{3}$ such that

$$
\Gamma_{1} \cap \Gamma_{2}=\varnothing .
$$

To formulate the problem, we need the following notation.

Let $\mathfrak{M}=\mathfrak{M}\left(\mathbb{R}^{3}\right)$ be the $\sigma$-algebra of Borel measures $\nu$ on $\mathbb{R}^{3}$, equipped with the vague topology, i. e., the topology of pointwise convergence on the class $C_{0}\left(\mathbb{R}^{3}\right)$ of all realvalued continuous functions on $\mathbb{R}^{3}$ with compact support (see, e.g., $[2,11]$ ). The mutual Newtonian energy of $\nu_{1}, \nu_{2} \in \mathfrak{M}$ is given by the formula

$$
I\left(\nu_{1}, \nu_{2}\right):=\iint_{\mathbb{R}^{3}} \int_{\mathbb{R}^{3}} \frac{d \nu_{1}(x) d \nu_{2}(y)}{|x-y|}
$$

(certainly, if the integral on the right is well defined - as a finite number or $\pm \infty$ ). For $\nu_{1}=\nu_{2}$ we get the energy $I\left(\nu_{2}\right):=I\left(\nu_{2}, \nu_{2}\right)$ of $\nu_{2}$, and for $\nu_{1}=\delta_{x}$ - the value of the potential of $\nu_{2}$ at $x \in \mathbb{R}^{3}$, denoted by $U_{\nu_{2}}(x)$; namely,

$$
U_{\nu}(x):=\int_{\mathbb{R}^{3}} \frac{d \nu(y)}{|x-y|}, \quad \text { where } \quad \nu \in \mathfrak{M} \quad \text { and } x \in \mathbb{R}^{3} .
$$

Let $\mathcal{E}=\mathcal{E}\left(\mathbb{R}^{3}\right)$ consist of all $\nu \in \mathfrak{M}\left(\mathbb{R}^{3}\right)$ with finite energy. Since the Newtonian kernel $|x-y|^{-1}$ is strictly positive definite (see, e.g., [11] and the references given there), 
the bilinear form $I\left(\nu_{1}, \nu_{2}\right)$ defines on $\mathcal{E}$ a scalar product. Hence, $\mathcal{E}$ can be treated as a pre-Hilbert space with the norm

$$
\|\nu\|_{\mathcal{E}}:=\sqrt{I(\nu)}
$$

Given a closed set $F \subset \mathbb{R}^{3}$, let $\mathfrak{M}(F)$ consist of all $\nu \in \mathfrak{M}\left(\mathbb{R}^{3}\right)$ supported by $F$, and let $\mathfrak{M}^{+}(F)$ be the convex cone of all non-negative $\nu \in \mathfrak{M}(F)$. We also write

$$
\mathcal{E}^{+}(F):=\mathfrak{M}^{+}(F) \cap \mathcal{E}
$$

The condenser $\mathcal{A}=\left(\Gamma_{1}, \Gamma_{2}\right)$ is supposed to be loaded by charges

$$
\mu=\mu^{1}-\mu^{2}, \quad \text { where } \mu^{j} \in \mathcal{E}^{+}\left(\Gamma_{j}\right), j=1,2 .
$$

The collection of all those $\mu$ will be denoted by $\mathcal{E}(\mathcal{A})$; it is a convex cone in $\mathcal{E}$.

Further, let $g$ be a given continuous, positive function on $\Gamma:=\Gamma_{1} \cup \Gamma_{2}$ and $a=\left(a_{1}, a_{2}\right) \in$ $\mathbb{R}^{2}$ a given vector with $a_{j}>0, j=1,2$. Then the set of admissible charges for the Gauss problem is given by

$$
\mathcal{E}(\mathcal{A}, a, g):=\left\{\mu \in \mathcal{E}(\mathcal{A}): \int_{\Gamma_{j}} g d \mu^{j}=a_{j}, j=1,2\right\} .
$$

Observe that $\mathcal{E}(\mathcal{A}, a, g)$ is an affine, convex cone in the pre-Hilbert space $\mathcal{E}$.

In addition, let $f$ denote a given continuous function on $\Gamma$, characterizing an exterior source of energy. Then

$$
\mathbb{G}_{f}(\mu):=I(\mu)-2 \int_{\Gamma} f d \mu
$$

defines the value of the so-called Gauss functional at $\mu \in \mathcal{E}(\mathcal{A})$.

The Gauss problem now reads:

Find $\mu_{0}$ that minimizes $\mathbb{G}_{f}(\mu)$ in $\mathcal{E}(\mathcal{A}, a, g)$, i. e., $\mu_{0} \in \mathcal{E}(\mathcal{A}, a, g)$ with

$$
\mathbb{G}_{f}\left(\mu_{0}\right)=\inf _{\mu \in \mathcal{E}(\mathcal{A}, a, g)} \mathbb{G}_{f}(\mu)=: \mathbb{G}_{f}(\mathcal{A}, a, g) .
$$

A minimizing measure $\mu_{0}$ is unique (if exists). This follows from the strict positive definiteness of the Newtonian kernel and the convexity of the class of admissible measures; see Lemma 6 in [22]. But what about the existence of $\mu_{0}$ ?

Assume for a moment that at least one of $\Gamma_{1}$ and $\Gamma_{2}$ is noncompact. Then it is not clear at all that the equilibrium state in the Gauss variational problem can be attained. Moreover, it has recently been shown by the third author that, in this case, a minimizing measure $\mu_{0}$ in general does not exist; necessary and sufficient conditions for $\mu_{0}$ to exist were given in [20, 21, 23].

However, in the case under consideration, where both $\Gamma_{1}$ and $\Gamma_{2}$ are assumed to be compact, the Gauss variational problem has a (unique) solution in the cone $\mathcal{E}(\mathcal{A}, a, g)$. Indeed, this follows from the vague compactness of $\mathcal{E}(\mathcal{A}, a, g)$ when combined with the fact that the Gauss functional $\mathbb{G}_{f}$ is vaguely lower semicontinuous on $\mathcal{E}(\mathcal{A})$. Cf. [14].

Remark: If $f=0, g=1$ and $\Gamma$ is only one connected manifold, then the potential $U_{\mu_{0}}(x)$ is constant for $x \in \Gamma$, and $d \mu_{0}$ is given by the so-called natural layer or Robin density (see $[9])$. 


\section{The variational formulation in a trace space}

In this section we show that for the compact plates $\Gamma_{1}, \Gamma_{2}$ given as two closed Lipschitz surfaces in $\mathbb{R}^{3}$, the Gauss problem (2.1) is equivalent to a variational problem in a particular Sobolev-Slobodetski space on $\Gamma=\Gamma_{1} \cup \Gamma_{2}$. Let $\Omega \subset \mathbb{R}^{3}$ be the domain (bounded or unbounded) with the boundary $\partial_{\mathbb{R}^{3}} \Omega=\Gamma$, and let $H^{\frac{1}{2}}(\Gamma)$ be the space of traces of the Sobolev space $H^{1}(\Omega)$ onto $\Gamma$ (see [1]).

Note that $\Gamma$ is Lipschitz. Let $C^{\infty}(\Gamma)$ be the trace space on $\Gamma$ of $C^{\infty}\left(\mathbb{R}^{3}\right)$, and define for $\varphi \in C^{\infty}(\Gamma)$

$$
\|\varphi\|_{H^{\frac{1}{2}(\Gamma)}}:=\inf \left\{\|\widetilde{\varphi}\|_{H^{1}(\Omega)} \text { where }\left.\widetilde{\varphi}\right|_{\Gamma}=\varphi\right\} .
$$

Then $C^{\infty}(\Gamma)$ is dense in the trace space $H^{\frac{1}{2}}(\Gamma)$, its closure with respect to the norm given by $(3.1)$ (see [1]).

Moreover, the surface measure $d s$ on $\Gamma$ is well defined and generates on $C^{\infty}(\Gamma)$ the $L_{2}$ scalar product,

$$
(\varphi, \psi):=\int_{\Gamma} \varphi \psi d s, \quad \text { where } \varphi, \psi \in C^{\infty}(\Gamma) .
$$

In fact, $H^{\frac{1}{2}}(\Gamma)$ is a Hilbert space equipped with the scalar product

$$
((\varphi, \psi))_{H^{\frac{1}{2}}(\Gamma)}:=(\varphi, \psi)+\int_{\Gamma} \int_{\Gamma} \frac{(\varphi(x)-\varphi(y))(\psi(x)-\psi(y))}{|x-y|^{3}} d s(x) d s(y)
$$

and the norms given by $(3.1)$ and by $((\varphi, \varphi))_{H^{\frac{1}{2}(\Gamma)}}^{\frac{1}{2}}$ are equivalent (see $\left.[1,7.48]\right)$.

The $L_{2}$ scalar product (3.2) continuously extends to the duality between $H^{\frac{1}{2}}(\Gamma)$ and its dual space $H^{-\frac{1}{2}}(\Gamma)$, which is equipped with the norm

$$
\|\varphi\|_{H^{-\frac{1}{2}(\Gamma)}}:=\sup \left\{|(\varphi, \psi)| \text { for } \psi \in H^{\frac{1}{2}}(\Gamma) \text { with }\|\psi\|_{H^{\frac{1}{2}(\Gamma)}} \leq 1\right\} .
$$

We denote that extension by the same symbol $(\cdot, \cdot)$. Since $\Gamma$ is Lipschitz, the function space $C^{\infty}(\Gamma)$ is dense in each of the spaces $H^{\frac{1}{2}}(\Gamma), L_{2}(\Gamma)$, and $H^{-\frac{1}{2}}(\Gamma)$.

For the Gauss variational problem (2.1) we shall find an equivalent formulation which is based on special distributions concentrated on $\Gamma$ with densities $\varphi \in H^{-\frac{1}{2}}(\Gamma)$. These define bounded linear functionals on $H^{\frac{1}{2}}(\Gamma) \supset C^{\infty}(\Gamma)$, whereas Borel measures $\mu \in \mathfrak{M}(\Gamma)$ define bounded linear functionals on $C(\Gamma)$; however, $C(\Gamma) \not \subset H^{\frac{1}{2}}(\Gamma) \not \subset C(\Gamma)$.

In $H^{-\frac{1}{2}}(\Gamma)$, let us define the affine cone

$$
\begin{gathered}
\mathcal{K}(\mathcal{A}, a, g):=\left\{\varphi=\varphi^{1}-\varphi^{2}, \quad \text { where } \operatorname{supp} \varphi^{j} \subset \Gamma_{j},\left.\varphi^{j}\right|_{\Gamma_{j}} \in H^{-\frac{1}{2}}\left(\Gamma_{j}\right),\right. \\
\left.\varphi^{j} \geq 0 \text { and } \int_{\Gamma_{j}} g \varphi^{j} d s=a_{j}, j=1,2\right\}
\end{gathered}
$$


where $a_{1}, a_{2}$ are the two given positive constants. From now on, for the given positive function $g$ we require $g \in C(\Gamma) \cap H^{\frac{1}{2}}(\Gamma)$. As we shall see, the solution of the Gauss problem (2.1) can be obtained with the help of the simple layer potential operator

$$
V \psi(x):=\int_{\Gamma} \frac{\psi(y)}{|x-y|} d s(y) \text { for } x \in \Gamma,
$$

which for $\psi \in H^{-\frac{1}{2}}(\Gamma)$ is well defined almost everywhere on the Lipschitz surface $\Gamma$ (see $[3,9,19])$.

Let us collect some of the properties of $V$, for Lipschitz surfaces $\Gamma$ proved by Costabel [3] and Verchota [19], in the following theorem.

Theorem 3.1 For every $\sigma \in\left[-\frac{1}{2}, \frac{1}{2}\right], V$ is a linear, continuous, invertible mapping

$$
V: H^{-\frac{1}{2}+\sigma}(\Gamma) \rightarrow H^{\frac{1}{2}+\sigma}(\Gamma) .
$$

Moreover, it is $H^{-\frac{1}{2}}(\Gamma)$-elliptic; i.e., there exist constants $c, c_{V}>0$ depending on $\Gamma$ only such that

$$
c\|\psi\|_{H^{-\frac{1}{2}(\Gamma)}}^{2} \geq\|\psi\|_{V}^{2} \geq c_{V}\|\psi\|_{H^{-\frac{1}{2}(\Gamma)}}^{2} \text { for all } \psi \in H^{-\frac{1}{2}}(\Gamma),
$$

where

$$
\|\psi\|_{V}^{2}:=(\psi, V \psi)
$$

Now we are in a position to characterize all the Borel measures of finite energy via distributions in $H^{-\frac{1}{2}}(\Gamma)$.

Theorem 3.2 Let $\Sigma \in \mathfrak{M}(\Gamma)$ have finite Newtonian energy

$$
I(\Sigma)=\|\Sigma\|_{\mathcal{E}}^{2}<\infty .
$$

Then there exists a unique element $\sigma \in H^{-\frac{1}{2}}(\Gamma)$ such that

$$
\Sigma(\phi)=\int_{\Gamma} \phi d \Sigma=(\phi, \sigma) \text { for all } \phi \in C^{\infty}(\Gamma) .
$$

Moreover,

$$
\|\Sigma\|_{\mathcal{E}}^{2}=\|\sigma\|_{V}^{2} \simeq\|\sigma\|_{H^{-\frac{1}{2}}(\Gamma)}^{2}
$$

where $\simeq$ denotes equivalence. We shall call $\sigma$ the distribution in $H^{-\frac{1}{2}}(\Gamma)$ associated with $\Sigma$.

Proof: Let $\phi \in C^{\infty}(\Gamma)$, then $\psi:=V^{-1} \phi \in L_{2}(\Gamma)$ since $C^{\infty}(\Gamma) \subset H^{1}(\Gamma)$ and

$$
\phi(x)=V \psi(x)=\int_{\Gamma} \frac{1}{|x-y|} \psi(y) d s(y)
$$


due to (3.7) for $\sigma=\frac{1}{2}$. Therefore,

$$
\Sigma(\phi)=\int_{\Gamma} \phi d \Sigma=\int_{\Gamma}\left(\int_{\Gamma} \frac{1}{|x-y|} \psi(y) d s(y)\right) d \Sigma(x)
$$

and with Fubini's theorem,

$$
\Sigma(\phi)=\int_{\Gamma}\left(\int_{\Gamma} \frac{1}{|x-y|} d \Sigma(x)\right) \psi(y) d s(y)=I(\Sigma, \Psi),
$$

where $\Psi$,

$$
\Psi(B):=\int_{B} \psi(y) d s(y) \text { for Borel sets } B \subset \Gamma,
$$

is a Borel measure on $\Gamma$ because $\psi \in L^{2}(\Gamma) \subset L^{1}(\Gamma)$. Moreover, since $L_{2}(\Gamma) \subset H^{-\frac{1}{2}}(\Gamma)$, we get

$$
\begin{aligned}
|I(\Psi, \Psi)| & =\left|\int_{\Gamma} \int_{\Gamma} \frac{1}{|x-y|} \psi(y) d s(y) \psi(x) d s(x)\right| \\
& =(\psi, V \psi) \leq\|V\| \psi\left\|_{H^{-\frac{1}{2}(\Gamma)}}^{2} \leq c_{0}^{2}\right\| \phi \|_{H^{\frac{1}{2}(\Gamma)}}^{2}<\infty,
\end{aligned}
$$

and the energy scalar product in (3.11) is thus well defined. Therefore,

$$
|\Sigma(\phi)|=|I(\Sigma, \Psi)| \leq\|\Sigma\|_{\mathcal{E}}\|\Psi\|_{\mathcal{E}} \leq c_{\Sigma} c_{0}\|\phi\|_{H^{\frac{1}{2}(\Gamma)}} \quad \text { for all } \phi \in C^{\infty}(\Gamma)
$$

where $c_{\Sigma}$ and $c_{0}$ are not depending on $\phi$. Since $C^{\infty}(\Gamma)$ is dense in $H^{\frac{1}{2}}(\Gamma), \Sigma$ defines a bounded linear functional on $H^{\frac{1}{2}}(\Gamma)$, and there exists a uniquely defined element $\sigma \in$ $H^{-\frac{1}{2}}(\Gamma)$ such that $(3.9)$ holds.

In order so show (3.10) we consider $\phi(y):=|x-y|^{-1}$ for any fixed $x \in \mathbb{R}^{3} \backslash \Gamma$ as a function of $y$. Then $\phi \in C^{\infty}(\Gamma)$ and

$$
\begin{aligned}
U_{\Sigma}(x) & =\int_{\Gamma} \frac{1}{|x-y|} d \Sigma(y)=\Sigma(\phi) \\
& =\int_{\Gamma} \frac{1}{|x-y|} \sigma(y) d s(y)=V \sigma(x)
\end{aligned}
$$

because of (3.9), and $V \sigma(x)$ here denotes the simple layer potential for $x \notin \Gamma$. This relation holds for every $x \notin \Gamma$, therefore the potentials $U_{\Sigma}$ and $V \sigma$ coincide everywhere in $\Omega$ as well as in $\Omega^{c}=\mathbb{R}^{3} \backslash \bar{\Omega}$. As is well known (see, e.g., [11, Th. 1.20]),

$$
\|\Sigma\|_{\mathcal{E}}^{2}=\frac{1}{4 \pi} \int_{\mathbb{R}^{3}}\left|\nabla U_{\Sigma}\right|^{2} d x .
$$


Hence, $\left.U_{\Sigma}\right|_{\Omega} \in H^{1}(\Omega)$ and $\left.U_{\Sigma}\right|_{\Omega^{c}} \in H^{1}\left(\Omega^{c}\right)$. Moreover, $U_{\Sigma}$ solves the following transmission problem (see [9, Sec. 5.6.3] and [18]):

$$
\begin{gathered}
\Delta U_{\Sigma}=0 \text { in } \Omega \cup \Omega^{c}, \\
\lim _{\Omega \ni x \rightarrow x_{0} \in \Gamma} U_{\Sigma}(x)=\lim _{\Omega^{c} \ni x \rightarrow x_{0} \in \Gamma} U_{\Sigma}(x) \text { for almost all } x_{0} \in \Gamma, \\
\left.\nabla U_{\Sigma}\right|_{\Omega} \cdot \mathbf{n}_{\partial \Omega}+\left.\nabla U_{\Sigma}\right|_{\Omega^{c}} \cdot \mathbf{n}_{\partial \Omega^{c}}=4 \pi \sigma \in H^{-\frac{1}{2}}(\Gamma) .
\end{gathered}
$$

In (3.13), almost everywhere means with respect to the surface measure on $\Gamma$ and the limits are supposed to be nontangential, while in (3.14) $\mathbf{n}_{\partial \Omega}$ and $\mathbf{n}_{\partial \Omega^{c}}$ denote the interior unit normal vectors with respect to $\Omega$ and $\Omega^{c}$, respectively, and the equality holds in the weak sense. Then it follows (see [3]) that

$$
(V \sigma, \sigma)=\frac{1}{4 \pi} \int_{\Omega}\left|\nabla U_{\Sigma}\right|^{2} d x+\frac{1}{4 \pi} \int_{\Omega^{c}}\left|\nabla U_{\Sigma}\right|^{2} d x,
$$

which is equivalent to $\|\sigma\|_{H^{-\frac{1}{2}}}^{2}$ and, furthermore, equals $\|\Sigma\|_{\mathcal{E}}^{2}$ due to (3.12). This completes the proof of Theorem 3.2.

Given $\varphi \in H^{-\frac{1}{2}}(\Gamma)$, write

$$
\mathbb{V}_{f}(\varphi):=\|\varphi\|_{V}^{2}-2(f, \varphi)
$$

As a consequence of Theorem 3.2, we conclude for the solution of the Gauss problem the following.

Theorem $3.3^{1}$ Let $f, g \in C(\Gamma) \cap H^{\frac{1}{2}}(\Gamma)$ and $g$ be strictly positive. Then the unique solution $\mu_{0} \in \mathcal{E}(\mathcal{A}, a, g)$ of the Gauss problem (2.1) defines a bounded linear functional on $H^{\frac{1}{2}}(\Gamma)$ and a unique element $\varphi_{0} \in \mathcal{K}(\mathcal{A}, a, g) \subset H^{-\frac{1}{2}}(\Gamma)$ with the properties

$$
\mu_{0}(\phi)=\left(\varphi_{0}, \phi\right) \text { for all } \phi \in C^{\infty}(\Gamma)
$$

and

$$
\mathbb{V}_{f}\left(\varphi_{0}\right)=\mathbb{G}_{f}\left(\mu_{0}\right)=\mathbb{G}_{f}(\mathcal{A}, a, g) .
$$

Moreover, $\varphi_{0}$ is the minimizer on $\mathcal{K}(\mathcal{A}, a, g)$ of the functional $\mathbb{V}_{f}$, i. e.,

$$
\mathbb{V}_{f}\left(\varphi_{0}\right)=\min _{\varphi \in \mathcal{K}(\mathcal{A}, a, g)} \mathbb{V}_{f}(\varphi)
$$

Proof: Since $\mathcal{A}$ is compact, there exists a unique solution $\mu_{0}$ of the Gauss problem in $\mathcal{E}(\mathcal{A}, a, g)$ (see Sec. 1). Hence, $I\left(\mu_{0}\right)<\infty$. Because of Theorem 3.2, there exists $\varphi_{0} \in H^{-\frac{1}{2}}(\Gamma)$ such that

$$
\mathbb{V}_{f}\left(\varphi_{0}\right)=\mathbb{G}_{f}\left(\mu_{0}\right)=\mathbb{G}_{f}(\mathcal{A}, a, g) .
$$

\footnotetext{
${ }^{1} \mathrm{~A}$ corresponding result for distributions is due to D. Medkova [12, Remark 7.11]
} 
Since $C^{\infty}(\Gamma)$ is dense in $H^{-\frac{1}{2}}(\Gamma)$ there exists a sequence $\varphi_{\ell} \in C^{\infty}(\Gamma) \cap \mathcal{K}(\mathcal{A}, a, g)$ converging to $\varphi_{0}$ in $H^{-\frac{1}{2}}(\Gamma)$. Therefore

$$
\mathbb{V}_{f}\left(\varphi_{\ell}\right) \rightarrow \mathbb{V}_{f}\left(\varphi_{0}\right)=\mathbb{G}_{f}(\mathcal{A}, a, g)
$$

Moreover, $\varphi d s \in \mathcal{E}(\mathcal{A}, a, g)$ for all $\varphi \in C^{\infty}(\Gamma) \cap \mathcal{K}(\mathcal{A}, a, g)$ and so

$$
\mathbb{G}_{f}(\mathcal{A}, a, g) \leq \inf _{\varphi \in \mathcal{K}(\mathcal{A}, a, g) \cap C^{\infty}(\Gamma)} \mathbb{V}_{f}(\varphi) \leq \mathbb{V}_{f}\left(\varphi_{\ell}\right) \text { for all } \ell \in \mathbb{N},
$$

which implies with $\ell \rightarrow \infty$

$$
\inf _{\varphi \in \mathcal{K}(\mathcal{A}, a, g) \cap C^{\infty}(\Gamma)} \mathbb{V}_{f}(\varphi)=\mathbb{V}_{f}\left(\varphi_{0}\right)=\mathbb{G}_{f}(\mathcal{A}, a, g)
$$

and (3.15) since $C^{\infty}(\Gamma)$ is dense in $H^{-\frac{1}{2}}(\Gamma)$.

In order to find a solution of the Gauss problem (2.1) with a suitable algorithm we replace the affine cone $\mathcal{K}(\mathcal{A}, a, g)$ by a cone $\mathcal{K}(\Gamma)$ with vertex at 0 by employing Lagrange multipliers for the side conditions $\int_{\Gamma_{j}} g \varphi_{j} d s=a_{j}, j=1,2$.

Then we arrive at the following problem: Find

$$
\begin{aligned}
\varphi_{\varrho} \in \mathcal{K}(\Gamma):= & \left\{\varphi \in H^{-\frac{1}{2}}(\Gamma), \text { where } \varphi=\varphi^{1}-\varphi^{2},\right. \\
& \left.\operatorname{supp} \varphi^{j} \subset \Gamma_{j},\left.\varphi^{j}\right|_{\Gamma_{j}} \in H^{-\frac{1}{2}}\left(\Gamma_{j}\right), \text { and } \varphi^{j} \geq 0\right\},
\end{aligned}
$$

which is the minimizer of

$$
\mathbb{V}_{f, \varrho}(\varphi):=\mathbb{V}_{f}(\varphi)+\frac{\varrho}{2} \sum_{j=1}^{2}\left|\int_{\Gamma_{j}} g \varphi^{j} d s-a_{j}\right|^{2}
$$

Namely,

$$
\mathbb{V}_{f, \varrho}\left(\varphi_{\varrho}\right)=\min _{\varphi \in \mathcal{K}(\Gamma)} \mathbb{V}_{f, \varrho}(\varphi)=: \mathbb{V}_{f, \varrho}(\mathcal{A}, a, g)
$$

Here $\varrho>0$ is a penalty parameter to be chosen later on appropriately.

Lemma 3.4 If $\varphi_{0} \in H^{-\frac{1}{2}}(\Gamma)$ defines the solution of the problem (2.1) due to Theorem 3.2, and $\varphi_{\varrho} \in H^{-\frac{1}{2}}(\Gamma)$ is the minimizer of (3.17), then we have

$$
\mathbb{V}_{f, \varrho}(\mathcal{A}, a, g) \leq \mathbb{G}_{f}(\mathcal{A}, a, g),
$$

and with

$$
C:=\frac{1}{c_{V}}\left\{c_{D}\|f\|_{H^{\frac{1}{2}(\Gamma)}}+\left[c_{D}^{2}\|f\|_{H^{\frac{1}{2}(\Gamma)}}^{2}+c_{V} \mathbb{G}_{f}(\mathcal{A}, a, g)\right]^{\frac{1}{2}}\right\}
$$


the a priori estimates for $\varphi_{0}$ and $\varphi_{\varrho}$ :

$$
\left\|\varphi_{0}\right\|_{H^{-\frac{1}{2}(\Gamma)}} \leq C \text { and }\left\|\varphi_{\varrho}\right\|_{H^{-\frac{1}{2}(\Gamma)}} \leq C
$$

where $c_{D}$ is the duality constant in

$$
|(\chi, \psi)| \leq c_{D}\|\chi\|_{H^{-\frac{1}{2}(\Gamma)}}\|\psi\|_{H^{\frac{1}{2}(\Gamma)}} .
$$

Proof: Since $\varphi_{0} \in \mathcal{K}(\mathcal{A}, a, g) \subset \mathcal{K}(\mathcal{A})$ and $\int_{\Gamma_{j}} g \varphi_{0}^{j} d s=a_{j}$ for $j=1$, 2, we get

$$
\begin{aligned}
\mathbb{V}_{f, \varrho}\left(\varphi_{\varrho}\right) & =\mathbb{V}_{f}\left(\varphi_{\varrho}\right)+\frac{\varrho}{2} \sum_{j=1}^{2}\left|\int_{\Gamma_{j}} g \varphi_{\varrho}^{j} d s-a_{j}\right|^{2} \\
& \leq \mathbb{V}_{f, \varrho}(\varphi)+0=\mathbb{G}_{f}(\mathcal{A}, a, g),
\end{aligned}
$$

i.e., (3.18). Then, with (3.8), we obtain

$$
\begin{aligned}
c_{V}\left\|\varphi_{\varrho}\right\|_{H^{-\frac{1}{2}(\Gamma)}}^{2} & \leq\left\|\varphi_{\varrho}\right\|_{V}^{2}=\mathbb{V}_{f, \varrho}(\mathcal{A}, a, g)+2\left(f, \varphi_{\varrho}\right)-\frac{\varrho}{2} \sum_{j=1}^{2}\left|\int_{\Gamma_{j}} g \varphi_{\varrho}^{j} d s-a_{j}\right|^{2} \\
& \leq \mathbb{G}_{f}(\mathcal{A}, a, g)+2 c_{D}\|f\|_{H^{\frac{1}{2}(\Gamma)}}\left\|\varphi_{\varrho}\right\|_{H^{-\frac{1}{2}(\Gamma)}} .
\end{aligned}
$$

So, with $C$ defined by (3.19) the second inequality in (3.20) follows.

For $\varphi_{0}$ we have the same estimate due to

$$
c_{V}\left\|\varphi_{0}\right\|_{H^{-\frac{1}{2}(\Gamma)}}^{2} \leq \mathbb{G}_{f}(\mathcal{A}, a, g)+2 c_{D}\|f\|_{H^{\frac{1}{2}(\Gamma)}}\left\|\varphi_{0}\right\|_{H^{-\frac{1}{2}(\Gamma)}} .
$$

Theorem 3.5 Let $\varphi_{0}$ be the minimizer of (2.1) and $\varphi_{\varrho}$ be the minimizer of (3.17). Then for

$$
\widetilde{\varphi}_{\varrho}^{j}=\alpha_{j} \varphi_{\varrho}^{j}, \quad \text { where } \quad \alpha_{j}^{-1}=a_{j}^{-1} \int_{\Gamma_{j}} \varphi_{\varrho}^{j} g d s,
$$

we find the following estimates:

$$
\left|\delta_{j}\right| \leq \delta \leq \sqrt{2} \varrho^{-\frac{1}{2}}\left\{\mathbb{G}_{f}(\mathcal{A}, a, g)+2 C\|f\|_{H^{\frac{1}{2}}(\Gamma)}\right\}=: C_{1} \varrho^{-\frac{1}{2}}
$$

where

$$
\delta_{j}:=\int_{\Gamma_{j}} \varphi_{\varrho}^{j} g d s-a_{j}, \quad \delta^{2}=\delta_{1}^{2}+\delta_{2}^{2},
$$

and

$$
\left\|\widetilde{\varphi}_{\varrho}-\varphi_{0}\right\|_{H^{-\frac{1}{2}(\Gamma)}} \leq \sqrt{C_{2} \delta} \leq C_{3} \varrho^{-\frac{1}{4}}
$$


where

$$
\begin{aligned}
& C_{2}=\frac{\sqrt{2}}{a_{0}}\left[19 C^{2}\|V\|+2 c_{D} C\|f\|_{H^{\frac{1}{2}(\Gamma)}}\right], \\
& C_{3}:=\left(C_{1} C_{2}\right)^{\frac{1}{2}}, \quad \text { and } a_{0}:=\min \left\{a_{1}, a_{2}\right\} .
\end{aligned}
$$

Here, $\|V\|$ denotes the norm of the operator $V: H^{-\frac{1}{2}}(\Gamma) \rightarrow H^{\frac{1}{2}}(\Gamma)$. Moreover,

$$
\begin{aligned}
\mathbb{V}_{f}(\mathcal{A}, a, g) \leq & \mathbb{G}_{f}(\mathcal{A}, a, g) \leq \mathbb{V}_{f}(\mathcal{A}, a, g)-\frac{\varrho^{2}}{2} \sum_{j=1}^{2} \delta_{j}^{2} \\
& +\sum_{j=1}^{2}\left\{\left(\alpha_{j}^{2}-1\right)\left(V \varphi_{\varrho}^{j}, \varphi_{\varrho}^{j}\right)-2\left(\alpha_{j}-1\right)\left(f, \varphi_{\varrho}^{j}\right)\right\} \\
& +\left(\alpha_{1} \alpha_{2}-1\right)\left\{\left(V \varphi_{\varrho}^{1}, \varphi_{\varrho}^{2}\right)+\left(V \varphi_{\varrho}^{2}, \varphi_{\varrho}^{1}\right)\right\} \\
\leq & \mathbb{V}_{f}(\mathcal{A}, a, g)+O\left(\varrho^{-\frac{1}{4}}\right) .
\end{aligned}
$$

The proof of Theorem 3.5 will be given in the Appendix.

Theorem 3.5 shows that the solution of the Gauss problem (2.1) can be approximated by the solutions of the minimizing problem (3.17) by choosing $\varrho$ in (3.17) large enough. The advantage of (3.17) lies in the fact that for (3.17) the cone $\mathcal{K}(\Gamma)$ is a cone with vertex 0 and not an affine cone as in (2.1). For solving (3.17) we shall use the gradient projection method to be described in Section 5 .

\section{The condenser problem}

This section deals with the case where $f=0, g=1$, and $a_{1}=a_{2}=1$. Observe that then $\mathbb{G}_{f}(\mathcal{A}, a, g)$, being equal to the minimum of the energy over the class of all $\mu \in \mathcal{E}(\mathcal{A})$ with $\mu\left(\Gamma_{j}\right)=1$ for $j=1,2$, is actually equal to $[\operatorname{cap} \mathcal{A}]^{-1}$, where cap $\mathcal{A}$ denotes the Newtonian capacity of the condenser $\mathcal{A}$. Hence,

$$
\Lambda:=\Lambda_{\mathcal{A}}:=[\operatorname{cap} \mathcal{A}] \mu_{0}
$$

is actually the Newtonian equilibrium measure on $\mathcal{A}$.

On the other hand, it follows from results of the third author (see $[20,24,26])$ that $\Lambda_{\mathcal{A}}$, the Newtonian equilibrium measure on $\mathcal{A}$, can also be obtained as the (unique) solution to the (dual) minimal energy problem over the class of all Borel measures $\nu$ of finite energy supported by $\Gamma$ such that

$$
U_{\nu}(x)= \begin{cases}C(\nu) & \text { for all } \quad x \in \Gamma_{1}, \\ C(\nu)-1 & \text { for all } x \in \Gamma_{2},\end{cases}
$$

where $C(\nu)$ is a constant depending on $\nu$. What is important is that the measures $\nu$, admissible in the dual variational problem, are no longer required to belong to $\mathcal{E}(\mathcal{A})$; that is, the measures $\left.\nu\right|_{\Gamma_{j}}, j=1,2$, are allowed to be signed.

In view of Theorem 3.2, this leads us to the following formulation in $H^{-\frac{1}{2}}(\Gamma)$. 
Theorem 4.1 Let $\lambda=\lambda^{1}-\lambda^{2} \in H^{-\frac{1}{2}}(\Gamma)$ with $\operatorname{supp} \lambda^{j} \subset \Gamma_{j}, j=1,2$, be the distribution associated with the Newtonian equilibrium measure $\Lambda=\Lambda_{\mathcal{A}}$. Then $\lambda$ is also the minimizer of the energy

$$
\|\sigma\|_{V}^{2}=\left(\sigma^{1}, V \sigma^{1}-V \sigma^{2}\right)+\left(\sigma^{2},-V \sigma^{1}+V \sigma^{2}\right)
$$

over the set $\mathcal{H}^{-\frac{1}{2}}(\Gamma)$ consisting of all $\sigma=\sigma^{1}-\sigma^{2} \in H^{-\frac{1}{2}}(\Gamma)$ with $\operatorname{supp} \sigma^{j} \subset \Gamma_{j}$ satisfying the system of boundary integral equations

$$
\begin{aligned}
& V \sigma^{1}-V \sigma^{2}=c \quad \text { on } \Gamma_{1}, \\
& -V \sigma^{1}+V \sigma^{2}=1-c \quad \text { on } \Gamma_{2} \text {, }
\end{aligned}
$$

where $c \in \mathbb{R}$ is a parameter.

Theorem 4.2 The distribution $\lambda=\lambda^{1}-\lambda^{2}$ associated with the Newtonian equilibrium measure $\Lambda=\Lambda_{\mathcal{A}}$ can be obtained with the following procedure:

Solve the system of boundary integral equations

$$
\begin{array}{ll}
V \dot{\sigma}^{1}-V \dot{\sigma}^{2}=1 & \text { on } \Gamma_{1}, \\
V \dot{\sigma}^{1}+V \dot{\sigma}^{2}=-1 & \text { on } \Gamma_{2}
\end{array}
$$

for $\dot{\sigma}=\dot{\sigma}^{1}-\dot{\sigma}^{2} \in H^{-\frac{1}{2}}(\Gamma)$, where $\operatorname{supp} \dot{\sigma}^{j} \subset \Gamma_{j}$. With

$$
C:=\left(\dot{\sigma}^{2}, 1\right) d^{-1} \in \mathbb{R}
$$

where

$$
d:=\left(\dot{\sigma}^{2}, 1\right)-\left(\dot{\sigma}^{1}, 1\right)
$$

solve the following system of boundary integral equations for $\lambda$ :

$$
\begin{aligned}
V \lambda^{1}-V \lambda^{2} & =C & \text { on } & \Gamma_{1}, \\
-V \lambda^{1}+V \lambda^{2} & =1-C & \text { on } & \Gamma_{2} .
\end{aligned}
$$

Then $\lambda=\lambda^{1}-\lambda^{2}$ is the desired solution.

Proof: Because of (4.3), the energy in (4.2) can also be written as

$$
\|\sigma\|_{V}^{2}=\left(\sigma^{1}, c\right)-\left(\sigma^{2}, c-1\right)
$$

where $\sigma^{1}, \sigma^{2}$ and the energy $\|\sigma\|_{V}^{2}$ depend on $c$, the constant value of the potential $V \sigma$ on $\Gamma_{1}$. The energy $\|\sigma\|_{V}^{2}$ becomes minimal if

$$
\begin{aligned}
\frac{\partial}{\partial c}\|\sigma\|_{V}^{2}=0 & =\left(\dot{\sigma}^{1}, c\right)+\left(\sigma^{1}, 1\right)-\left(\dot{\sigma}^{2}, c-1\right)-\left(\sigma^{2}, 1\right) \\
& =c\left[\left(\dot{\sigma}^{1}, 1\right)-\left(\dot{\sigma}^{2}, 1\right)\right]+\left(\dot{\sigma}^{2}, 1\right)+\left(\sigma^{1}, 1\right)-\left(\sigma^{2}, 1\right)
\end{aligned}
$$


where $\dot{\sigma}^{j}:=\partial \sigma^{j} / \partial c$.

Since the solution $\lambda$ of the minimum problem in Theorem 4.1 is the distribution associated with the Newtonian equilibrium measure $\Lambda_{\mathcal{A}}, \lambda$ satisfies the conditions

$$
\left(\lambda^{j}, 1\right)=\operatorname{cap} \mathcal{A} \quad \text { for } j=1,2,
$$

the last two terms in (4.7) cancel each other and the equation (4.7) now reads

$$
-c d+\left(\dot{\sigma}^{2}, 1\right)=0,
$$

which gives the required value of $C$.

Taking at $C$ the derivatives $\partial / \partial c$ of the solution of the minimum problem, we find

$$
\begin{aligned}
V \dot{\lambda}^{1}-V \dot{\lambda}^{2} & =1 \text { on } \Gamma_{1}, \\
-V \dot{\lambda}^{1}+V \dot{\lambda}^{2} & =-1 \quad \text { on } \Gamma_{2},
\end{aligned}
$$

i.e., system (4.4). Since the simple layer potential operator $V$ on $\Gamma_{1} \times \Gamma_{2}$ is strongly elliptic (see $[3,9]$ ), the system (4.4) has a unique solution $\dot{\lambda} \in H^{-\frac{1}{2}}(\Gamma)$. Then $C$ is uniquely determined provided $d \neq 0$, and with $c:=C$ we obtain $\lambda$ as the (unique) solution of the strongly elliptic boundary integral equation system (4.6).

Lemma 4.3 The constant $d=\left(\dot{\lambda}^{2}, 1\right)-\left(\dot{\lambda}^{1}, 1\right)$ is negative.

Proof: Without loss of generality, we can assume $\Gamma_{2}$ to contain boundary limit points of the unbounded component $\Omega_{\infty}$ of $\mathbb{R}^{3} \backslash \Gamma$, for if not, we replace the roles of $\Gamma_{1}$ and $\Gamma_{2}$. Let us consider the simple layer potential $U_{\dot{\lambda}}$ in $\mathbb{R}^{3} \backslash \Gamma$, which has boundary values almost everywhere on $\Gamma$. Then we conclude from (4.4) that

$$
U_{\dot{\lambda}}=1 \quad \text { a. e. on } \Gamma \text {. }
$$

Since $U_{\dot{\lambda}} \in H^{-\frac{1}{2}}(\Gamma)$, the normal derivative $\partial U_{\dot{\lambda}} / \partial \mathbf{n}$ exists almost everywhere on $\Gamma$ and belongs to $H^{-\frac{1}{2}}(\Gamma)$ (see [19]). Here, $\mathbf{n}$ denotes the interior normal vector with respect to $\Omega_{\infty}$. With the jump relation of the normal derivative of the simple layer potential $U_{\dot{\lambda}}$ across $\Gamma$ (see [3]) and since $U_{\dot{\lambda}}(x)=1$ for all $x$ in the bounded components of $\mathbb{R}^{3} \backslash \Gamma$, we find

$$
\frac{\partial U_{\dot{\lambda}}}{\partial \mathbf{n}}=-\dot{\lambda}^{1} \quad \text { on } \quad \Gamma_{1}, \quad \frac{\partial U_{\dot{\lambda}}}{\partial \mathbf{n}}=\dot{\lambda}^{2} \quad \text { on } \Gamma_{2} .
$$

Since $U_{\dot{\lambda}}(x)=O\left(|x|^{-1}\right)$ for $|x| \rightarrow \infty$, Green's theorem can be applied to $U_{\dot{\lambda}}$ in $\Omega_{\infty}$, which yields

$$
d=\left(\dot{\lambda}^{2}, 1\right)-\left(\dot{\lambda}^{1}, 1\right)=\left(\frac{\partial U_{\dot{\lambda}}}{\partial \mathbf{n}}, U_{\dot{\lambda}}\right)=-\int_{\Omega_{\infty}}\left|\nabla U_{\dot{\lambda}}\right|^{2} d x<0,
$$

since $U_{\dot{\lambda}}$ is not constant in $\Omega_{\infty}$. 


\section{The approximation of the Gauss problem by the use of piecewise constant charges}

Since the minimizing problem (3.17) is quadratic, one could compute the minimizer by using the gradient-projection method:

$$
\varphi_{k+1}:=P_{\mathcal{K}}\left(\varphi_{k}-\eta \mathbb{V}_{f, \varrho}^{\prime} \varphi_{k}\right), k=0,1, \ldots
$$

where $P_{\mathcal{K}}$ is the $H^{-1 / 2}(\Gamma)$-orthogonal projection onto $\mathcal{K}(\Gamma)$ (see $[6, \mathrm{I}(3.13)]$ ), and $\mathbb{V}_{f, \varrho}^{\prime}$ the Frechet derivative of the functional $\mathbb{V}_{f, \varrho}$ in (3.17). But a numerical realization of $P_{\mathcal{K}}$ is as difficult as solving the original problem (3.17) itself. Therefore we shall apply a gradientprojection iteration to the discretized formulations instead.

For solving (3.17) numerically, let us use a quasiregular family of triangulations $\mathcal{T}_{h}$ of the surface $\Gamma=\Gamma_{1} \cup \Gamma_{2}$ (see [17, Chap.10]), where $h$ denotes the maximum mesh width of the elements in $\mathcal{T}_{h}$. On the triangulation, we introduce piecewise constant functions $\mathcal{S}_{h}^{0}(\Gamma) \subset L_{2}(\Gamma) \subset H^{-1 / 2}(\Gamma)$ as the trial as well as the test space. Correspondingly, we define

$$
\mathcal{K}_{h}(\Gamma):=\left\{\varphi_{h} \in \mathcal{S}_{h}^{0}(\Gamma) \text { where } \varphi_{h}=\varphi_{h}^{1}-\varphi_{h}^{2} \text { with } \operatorname{supp} \varphi_{h}^{j} \subset \Gamma_{j} \text { and } \varphi_{h}^{j} \geq 0\right\} .
$$

The finite-dimensional approximation to (3.4) then reads:

Find the minimizer $\varphi_{\varrho h} \in \mathcal{K}_{h}(\Gamma)$ of the quadratic functional

$$
\begin{aligned}
\mathbb{V}_{f, \varrho}\left(\varphi_{h}\right):= & \left\{\left(V \varphi_{h}^{1}, \varphi_{h}^{1}\right)-\left(V \varphi_{h}^{1}, \varphi_{h}^{2}\right)-\left(V \varphi_{h}^{2}, \varphi_{h}^{1}\right)+\left(V \varphi_{h}^{2}, \varphi_{h}^{2}\right)\right. \\
& -2\left(f, \varphi_{h}^{1}\right)+2\left(f, \varphi_{h}^{2}\right) \\
& \left.+\frac{\varrho}{2}\left(\left(\int_{\Gamma_{1}} g \varphi_{h}^{1} d s-a_{1}\right)^{2}+\left(\int_{\Gamma_{2}} g \varphi_{h}^{2} d s-a_{2}\right)^{2}\right)\right\} \text { on } \varphi_{h} \in \mathcal{K}_{h}(\Gamma) .
\end{aligned}
$$

If $M$ denotes the number of elements in $\mathcal{T}_{h}$, which here is also the dimension of $\mathcal{S}_{h}^{0}$, then minimizing (5.3) on $\mathcal{K}_{h}(\Gamma)$ defines a quadratic programming problem with $M$ linear constraints given by (5.2). Since the bilinear form

$$
\mathbb{V}_{f, \varrho}(\varphi)=\|\varphi\|_{V}^{2}+\frac{\varrho}{2}\left(\left(g, \varphi^{1}\right)^{2}+\left(g, \varphi^{2}\right)^{2}\right)
$$

in $(5.3)$ is $H^{-\frac{1}{2}}(\Gamma)$-elliptic Theorem 5.2 in [6] implies convergence.

Lemma 5.1 For any $\varrho \geq 0$ fixed, the solutions $\varphi_{\varrho h}$ of (5.3) converge with $h \rightarrow 0$ strongly in $H^{-\frac{1}{2}}(\Gamma)$ to $\varphi_{\varrho}$, the minimizer of $\mathbb{V}_{f, \varrho}$ in $(3.17)$.

Because $M$, in general, is rather large, standard methods for finding the discrete minimizer of (5.3) requires long computing times (see [7], where just a few hundred degrees 
of freedom were used). On the other hand, (5.3) is of the type (3.8) in [6, Chap.I]. We therefore write the Frechet derivative of (5.3) operating on $\varphi_{\varrho h} \in \mathcal{S}_{h}^{0}$ in variational form:

$$
\begin{aligned}
\mathbb{V}_{f, \varrho}^{\prime}=2[ & \left(V \varphi_{\varrho h}^{1}, \chi_{h}^{1}\right)-\left(V \varphi_{\varrho h}^{1}, \chi_{h}^{2}\right)-\left(V \varphi_{\varrho h}^{2}, \chi_{h}^{1}\right)+\left(V \varphi_{\varrho h}^{2}, \chi_{h}^{2}\right) \\
& +\frac{\varrho}{2}\left(g, \varphi_{\varrho h}^{1}\right) \cdot\left(g, \chi_{h}^{1}\right)+\frac{\varrho}{2}\left(g, \varphi_{\varrho h}^{2}\right) \cdot\left(g, \chi_{h}^{2}\right) \\
- & \left.\left(f, \chi_{h}^{1}\right)+\left(f, \chi_{h}^{2}\right)-\frac{\varrho}{2} a_{1}\left(g, \chi_{h}^{1}\right)-\frac{\varrho}{2} a_{2}\left(g, \chi_{h}^{2}\right)\right]
\end{aligned}
$$

for all $\chi_{h}^{1} \in S_{h}^{0}\left(\Gamma_{1}\right), \chi_{h}^{2} \in S_{h}^{0}\left(\Gamma_{2}\right)$.

This Frechet derivative can be realized by using a multipole approximation (see [13]) and is equivalent to a linear system

$$
\mathfrak{A} \mathbf{x}-\mathcal{F}=\mathbf{0} \in \mathbb{R}^{M}
$$

of $M$ equations on $M$ variables collected in the vector $\mathbf{x} \in \mathbb{R}^{M}$, the coefficients of $\varphi_{0 h}^{1}-\varphi_{0 h}^{2}$ with respect to the corresponding basis defined by the characteristic functions of $\tau_{\ell} \in$ $\mathcal{T}_{h}, \ell=1, \ldots, M$.

Inserting the representations of $\varphi_{h}^{1}-\varphi_{h}^{2}$ with the coefficients $\mathbf{x}$ into (5.3), the discretized minimizing problem now reads:

Minimize

$$
\begin{gathered}
\mathbf{x}^{\top} \mathfrak{A}_{0} \mathbf{x}-2 \mathbf{f}^{\top} \mathbf{x}+\frac{\varrho}{2}\left\{\left(\mathbf{g}_{1}^{\top} \mathbf{x}-a_{1}\right)^{2}+\left(\mathbf{g}_{2}^{\top} \mathbf{x}-a_{2}\right)^{2}\right\} \\
\text { on } \mathbf{x} \in \mathbb{R}_{+}^{M} \text { where } \mathbb{R}_{+}^{M}=\left\{\mathbf{y} \in \mathbb{R}^{M} \mid y_{j} \geq 0, j=1, \ldots, M\right\} .
\end{gathered}
$$

Here $\mathfrak{A}_{0}=\left(\begin{array}{cc}V_{11, h} & -V_{12, h} \\ -V_{21, h} & V_{22, h}\end{array}\right)$ is the Galerkin matrix of $V$ on $\Gamma_{1} \times \Gamma_{2}$.

But this problem is a problem in $\mathbb{R}^{M}$ with a positive definite symmetric matrix $\mathfrak{A}$ and, hence, of the type (3.8) in [6]. Therefore, we now may use the gradient projection method $\left[6\right.$, Chap.I (3.12)] in the Eucledian space $\mathbb{R}^{M}$ :

$$
\begin{aligned}
& \widetilde{\mathbf{x}}^{k+1}:=\mathbf{x}^{k}-\eta\left(\mathfrak{A}_{0} \mathbf{x}^{k}+\frac{\varrho}{2} \mathbf{g} \mathbf{g}^{\top} \mathbf{x}^{k}-\mathcal{F}\right) \\
& \mathbf{x}^{k+1}:=P_{\mathbb{R}_{+}^{M}} \widetilde{\mathbf{x}}^{k+1}, k=0,1, \ldots
\end{aligned}
$$

where $\eta$ is appropriately chosen, $0<\eta<2 \gamma_{h} /\|\mathfrak{A}\|_{\mathbb{R}^{M}, \mathbb{R}^{M}}^{2}, \mathbf{g}=\left(\begin{array}{l}\mathbf{g}_{1} \\ \mathbf{g}_{2}\end{array}\right)$ and $\mathcal{F}=\mathbf{f}+$ $\frac{\varrho}{2}\left(a_{1} \mathbf{g}_{1}^{\top}, a_{2} \mathbf{g}_{2}^{\top}\right)^{\top}$, and $\|\mathfrak{A}\|_{L_{2}, L_{2}}$ the spectral norm. The full matrix $\mathfrak{A}=\mathfrak{A}_{0}+\frac{\varrho}{2} \mathbf{g}\left(\mathbf{g}_{1}^{\top}, \mathbf{g}_{2}^{\top}\right) \in$ $\mathbb{R}^{M} \times \mathbb{R}^{M}$ satisfies a coerciveness inequality

$$
\mathbf{x}^{\top} \mathfrak{A} \mathbf{x} \geq \gamma_{h}\|\mathbf{x}\|_{\mathbb{R}^{M}}^{2} \text { for all } \mathbf{x} \in \mathbb{R}^{M}
$$

with

$$
\gamma_{h}=c_{V} \cdot c_{0} \cdot h>0
$$


The projection $P_{\mathbb{R}_{+}^{M}}$ is given by

$$
\left(P_{\mathbb{R}_{+}^{M}} \mathbf{y}\right)_{j}:= \begin{cases}y_{j} & \text { if } y_{j} \geq 0 \text { and } \\ 0 & \text { if } y_{j}<0 .\end{cases}
$$

Obviously,

$$
\left\|P_{\mathbb{R}_{+}^{M}}\right\|_{L_{2}\left(\mathbb{R}^{M}\right), L_{2}\left(\mathbb{R}^{M}\right)}=1
$$

and, therefore, (5.7) converges in $\mathbb{R}^{M}$ as the geometric series $\sum_{k=0}^{\infty}(1-\eta)^{k}$.

Although all matrix times vector multiplications are executed with the fast multipole approximation [13], because of (5.9) and as will be seen from the computations, the convergence turns out to be rather slow.

\subsection{Some computations with the projection iteration}

As computational examples, we consider a sphere for $\Gamma_{1}$ and for $\Gamma_{2}$ a rotational symmetric surface with radius $R\left(x_{1}\right)$ decaying to zero for $x_{1} \rightarrow \infty$. The surfaces are given by

$$
\begin{aligned}
\Gamma_{1}= & \left\{x \in \mathbb{R}^{3} \quad \text { with }|x+(2,0,0)|=1\right\} \\
\Gamma_{2}= & \left\{x \in \mathbb{R}^{3} \quad \text { with } x_{2}^{2}+x_{3}^{2} \leq 1 \text { for } x_{1}=0, \quad x_{2}^{2}+x_{3}^{2}=R\left(x_{1}\right),\right. \\
& \text { for } \left.0<x_{1}<X, \quad x_{2}^{2}+x_{3}^{2} \leq R(X) \text { for } x_{1}=X\right\} .
\end{aligned}
$$

In particular, we choose

$$
R(r)=(1+r)^{-1}
$$

The infinite rotational body is cut off at $X=4$.

For first tests of the gradient-projection method, we consider the Gauss problem (2.1) with $g=0.1, f(x)=1000\left|x-x_{0}\right|^{-1}$ where $x_{0}=(-4.0,0,0)^{\top}$, and use an approximation of $\Gamma_{1}$ and $\Gamma_{2}(X=4)$ with 2048 and 2088 triangles, respectively, see Fig. 1. We computed the charges $a_{1}=848.8032$ and $a_{2}=564.4113$ for $\varrho=0$ and then tried to recover the charges for several values of $\varrho \in\{1,10,100,1000\}$ by employing the gradient-projection iteration (5.7). We observed that $\eta$ decreases rapidly for large $\varrho$ and hence, a very slow convergence. In addition, the residual is still large after millions of iteration steps for large $\varrho$, but at the same time we observed a bad approximation of the charge distribution.

Next, we checked the convergence of the approximate total charges $\tilde{a}_{1}$ and $\tilde{a}_{2}$ for $\varrho \in$ $\{0,10,100,1000\}$ in the case of active constraints for chosen values of $a_{1}=400$ and $a_{2}=$ 300. In Tab. 1, we observe convergence of the computed approximations to $a_{1}$ and $a_{2}$ for increasing $\varrho$, which was proposed in (3.24). A relative accuracy of $10^{-4}$ was used as stopping criterion in the gradient-projection method. For $\varrho=1000$, however, the iteration was stopped after $3 \cdot 10^{6}$ steps. 


\begin{tabular}{r|r|r|r|r}
$\varrho$ & 0 & 10 & 100 & 1000 \\
\hline$\tilde{a}_{1}$ & 848.94 & 640.18 & 448.63 & 405.44 \\
$\tilde{a}_{2}$ & 564.67 & 408.89 & 314.04 & 301.34
\end{tabular}

Table 1: Approximations $\tilde{a}_{1}$ and $\tilde{a}_{2}$ of $a_{1}$ and $a_{2}$ of the gradient-projection method for the Gauss variational problem with $a_{1}=400 a_{2}=300 \mathrm{~g}=0.1$.

Since the charges converge so slowly for large $\varrho$, we now use a cascading approach. We start with small values of $\varrho$ to compute a good approximation of the charge distribution with a few iteration steps. Then this approximation serves as an initial guess for a new run of the gradient-projection method with a larger $\varrho$ to improve the approximation of the total charges. In each run, we limit the maximal number of iteration steps. Repeating this approach several times, we get a good approximation with a significantly smaller total number of iteration steps.

\begin{tabular}{r|r|r|r} 
& $\tilde{a}_{1}$ & $\tilde{a}_{2}$ & functional \\
\hline gradient proj. method & 405.4389 & 301.3350 & $-4.909957 \cdot 10^{6}$ \\
cascading approach & 405.4682 & 301.3556 & $-4.935061 \cdot 10^{6}$
\end{tabular}

Table 2: Results by the pure gradient-projection and by cascading for the Gauss variational problem with $g=0.1, a_{1}=400, a_{2}=300$.

For our example, we choose $\varrho \in\{10,50,100,300,500,700,800,900,950,1000\}$, limit the number of iteration steps in each run to 30000 and compare the results with the results of the gradient-projection method for $\varrho=1000$ and $3 \cdot 10^{6}$ iterations. In Table 2, we see a good agreement of the values $\tilde{a}_{1}$ and $\tilde{a}_{2}$ of the gradient-projection method and the cascading approach with a total of $3 \cdot 10^{5}$ iterations. In addition, the functional of the approximate solution of the cascading approach is smaller. This is reflected even better in Fig. 1. Altogether we get a much better approximation of the charge distribution by the cascading approach than with the pure gradient-projection method.
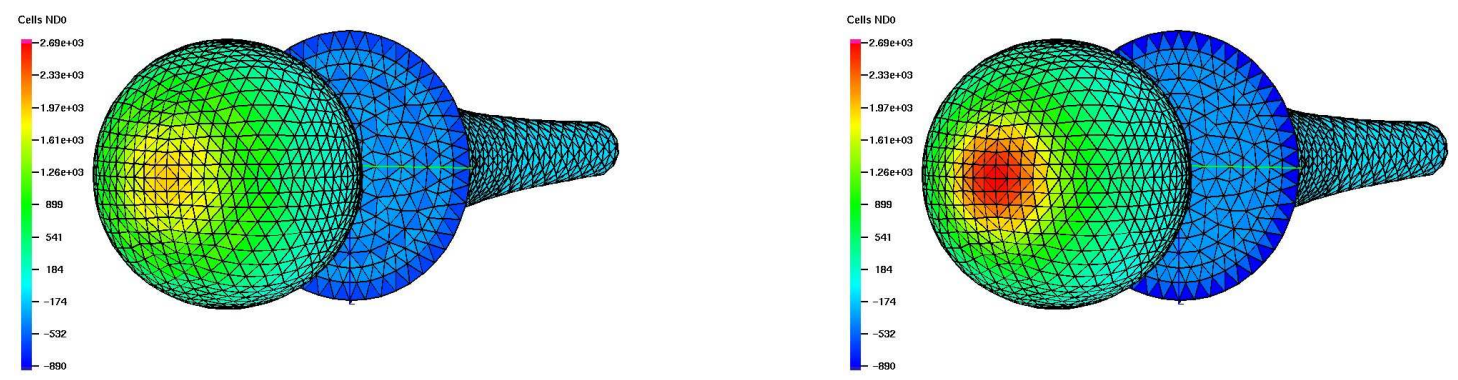

Figure 1: Approximate solution for $\varrho=1000$ and the Problem (5.3) $\left(a_{1}=400 a_{2}=300\right.$ $g=0.1)$ computed by the gradient-projection method and by cascading. 


\section{The approximation of the condenser problem}

For the numerical solution of the two systems (4.4) and (4.6) with (4.5) we use the same boundary triangulations $\mathcal{T}_{h}$ of $\Gamma=\Gamma_{1} \cup \Gamma_{2}$ as in Section 5. But now as test and trial functions in the Galerkin approximation we employ the full space $\mathcal{S}_{h}^{0}$ and set

$$
\sigma_{h}=\left(\sigma_{h}^{1}-\sigma_{h}^{2}\right) \text { where } \sigma_{h}^{j}=\sum_{\ell \in I_{j}} x_{\ell} \chi_{h \ell}, x_{\ell} \in \mathbb{R}
$$

with $\chi_{h \ell}$ the characteristic function to the triangle $\tau_{\ell} \in \mathcal{T}_{h} \cap \Gamma_{j}$ where $I_{1}=\left\{1, \ldots, M_{1}\right\}$ for the triangulation of $\Gamma_{1}$ and $I_{2}=\left\{M_{1}+1, \ldots, M\right\}$ for the triangulation of $\Gamma_{2}$. With the Galerkin matrix $\mathfrak{A}_{0}$ of $V$ on $\Gamma_{1} \times \Gamma_{2}$, the Galerkin equations to (4.4) now are equivalent to the linear system

$$
\mathfrak{A}_{0} \dot{\mathbf{x}}=\mathbf{g}
$$

for the coefficients $\dot{\mathbf{x}}$ of

$$
\dot{\sigma}_{h}^{1}=\sum_{\ell \in I_{1}} \dot{x}_{\ell} \chi_{h \ell} \text { on } \Gamma_{1} \text { and } \dot{\sigma}_{h}^{2}=\sum_{\ell \in I_{2}} \dot{x}_{\ell} \chi_{h \ell} \text { on } \Gamma_{2} .
$$

Then the approximations of $d$ in (4.5) and $C$ are obtained by solving the Galerkin equations of (4.6), i.e., the additional linear system

$$
\mathfrak{A}_{0} \mathbf{x}=\mathbf{u}
$$

for $\mathbf{x}$, and

$$
\lambda_{h}=\lambda_{h}^{1}-\lambda_{h}^{2} \text { with } \lambda_{h}^{1}=\sum_{\ell \in I_{1}} x_{\ell} \chi_{h \ell}, \lambda_{h}^{2}=\sum_{\ell \in I_{2}} x_{\ell} \chi_{h \ell},
$$

which is the desired approximation.

The solutions of the linear systems (6.2) and (6.3) are obtained with a preconditioned conjugate gradient method as in [13] where the preconditioner to (6.2) as well as to (6.3) is based on an artificial multilevel approach due to Steinbach in [16].

The corresponding matrix times vector multiplications are executed by using the fast multipole method [13] for the simple layer potential operator.

Since for this condenser problem the two linear systems (4.4) and (4.6) in $H^{-\frac{1}{2}}(\Gamma)$ are approximately solved by Galerkin's method, standard a priori error estimates for $h \rightarrow 0$ are available [8].

Lemma 6.1 Let $\dot{\lambda}, \lambda \in H^{s}(\Gamma)$ with $-\frac{1}{2} \leq s \leq 1$, and $-1-s \leq t \leq s \leq 1$, and let the family of triangulations be quasiregular. Then there hold the asymptotic a priori estimates

$$
\begin{aligned}
\left\|\dot{\lambda}_{h}-\dot{\lambda}\right\|_{H^{t}(\Gamma)} & \leq c h^{s-t}\|\dot{\lambda}\|_{H^{s}(\Gamma)},\left\|\lambda_{h}-\lambda\right\|_{H^{t}(\Gamma)} \leq c h^{s-t}\|\dot{\lambda}\|_{H^{s}(\Gamma)}, \\
\left|C_{h}-C\right| & \leq c h^{2 s+1} \text { and }\left|(\operatorname{cap} \mathcal{A})_{\mathrm{h}}-\operatorname{cap} \mathcal{A}\right| \leq \mathrm{ch}^{2 \mathrm{~s}+1} .
\end{aligned}
$$

If the family of triangulations is not quasiregular then (6.4) holds only for $-1-s \leq t \leq$ $-\frac{1}{2} \leq s \leq 1$.

Since the constant right-hand sides in (4.4) and (4.6) are in $C^{\infty}(\Gamma)$, the regularity, i.e. $s$ depends only on the regularity of $\Gamma$. 


\subsection{Some computations for the condenser problem}

For the computational examples of the condenser problem (4.4) and (4.6) with (4.5), we choose $a_{1}=1, a_{2}=1, f=0$, and $g=1$.

In Table 3, we compare the solutions of the condenser problem for several levels of discretization of the two bodies, which were considered in Sect. 5.1 and are given by (5.11) and (5.12) for $X=4$. The meshes are created by uniform refinement of the coarsest mesh with 308 triangles, where the new vertices are projected onto $\Gamma_{1}$ and $\Gamma_{2}$. We consider the approximate solution $\lambda_{7}$ on the finest refinement level with about 5 million triangles as a reference solution and compute an indicated error as

$$
\text { error }:=\left\|\lambda_{L}-\lambda_{7}\right\|_{L_{2}(\Gamma)} /\left\|\lambda_{7}\right\|_{L_{2}(\Gamma)} .
$$

We observe a reduction of the error but a low order of convergence eoc as predicted in (6.4). But the convergence is a lot better for the approximation of the charges at the tip of $\Gamma_{2}$, which is of further interest in this example.

\begin{tabular}{r|r|r|r|r}
$\mathrm{L}$ & $M$ & error & eoc & density \\
\hline 0 & 308 & 0.392 & 0.107 & -1.510673 \\
1 & 1232 & 0.364 & 0.128 & -1.445448 \\
2 & 4928 & 0.333 & 0.153 & -1.429619 \\
3 & 19712 & 0.299 & 0.184 & -1.424071 \\
4 & 78848 & 0.264 & 0.242 & -1.421334 \\
5 & 315392 & 0.223 & 0.242 & -1.419588 \\
6 & 1261568 & 0.168 & 0.405 & -1.418401 \\
7 & 5046272 & & & -1.417603
\end{tabular}

Table 3: Convergence study for $X=4$.

The charges at the tip of the rotational body $\Gamma_{2}$ are defined by the integral

$$
\int_{x \in \Gamma_{2}: x_{1}>X-10^{-8}} \lambda_{h}(x) d s_{x}
$$

for increasing values of the length $X$. For these computations, we use highly adaptive meshes with 50240 up to 446372 triangles for the approximation of $\Gamma_{1}$ and $\Gamma_{2}$. In Table 4, the charges at the tips of $\Gamma_{2}$ are devided by the areas at the tips for several $X$. In addition, the computed densities, which are given by the quotients of the charges and the areas of the tips, indicate the existence of a limit, which was proven to exist in [20].

With these computations, however, we reach the limits of the currently available software based on the fast and data-sparse realization of the single layer potential by the fast multipole method. For larger $X$, additional techniques will be necessary such as, e.g., appropriate domain decomposition methods. 


\begin{tabular}{l|r|r|r|r|r|r} 
& $X=8$ & $X=16$ & $X=24$ & $X=32$ & $X=40$ & $X=48$ \\
\hline $\int_{x_{1}>a} \lambda_{h} d s_{x}$ & $-7.20 \mathrm{e}-2$ & $-2.57 \mathrm{e}-2$ & $-1.33 \mathrm{e}-2$ & $-8.26 \mathrm{e}-3$ & $-5.61 \mathrm{e}-3$ & $-4.07 \mathrm{e}-3$ \\
$\int_{x_{1}>a} 1 d s_{x}$ & $3.88 \mathrm{e}-2$ & $1.09 \mathrm{e}-2$ & $5.02 \mathrm{e}-3$ & $2.88 \mathrm{e}-3$ & $1.87 \mathrm{e}-3$ & $1.31 \mathrm{e}-3$ \\
density & -1.86 & -2.36 & -2.65 & -2.87 & -3.00 & -3.11 \\
\hline & $X=56$ & $X=64$ & $X=72$ & $X=80$ & $X=88$ & $X=104$ \\
\hline $\int_{x_{1}>a} \lambda_{h} d s_{x}$ & $-3.11 \mathrm{e}-3$ & $-2.45 \mathrm{e}-3$ & $-1.98 \mathrm{e}-3$ & $-1.65 \mathrm{e}-3$ & $-1.39 \mathrm{e}-3$ & $-1.02 \mathrm{e}-3$ \\
$\int_{x_{1}>a} 1 d s_{x}$ & $9.66 \mathrm{e}-4$ & $7.43 \mathrm{e}-4$ & $5.89 \mathrm{e}-4$ & $4.78 \mathrm{e}-4$ & $3.96 \mathrm{e}-4$ & $2.85 \mathrm{e}-4$ \\
density & -3.21 & -3.30 & -3.36 & -3.45 & -3.51 & -3.57 \\
\hline
\end{tabular}

Table 4: Charges (6.5) and their densities at the tips for $a=X-10^{-8}$

As a second example, we consider again two bodies given by (5.11), where now

$$
R(r)=e^{-r} \quad \text { for } r \geq 0 .
$$

The solution for a discretization with 82364 boundary elements for $X=4$ is given in Fig. 2 . The solution is positive on $\Gamma_{1}$ and negative on $\Gamma_{2}$ as required.
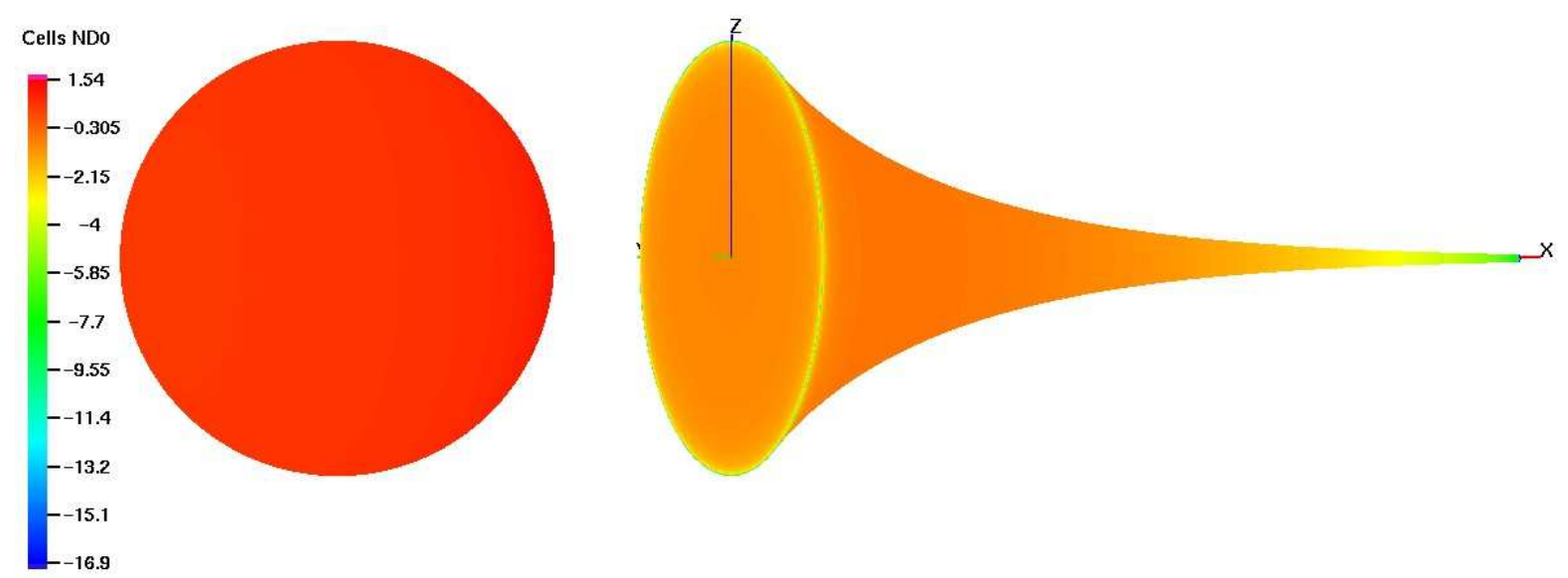

Figure 2: Charge density for 32768 and 49596 elements for $X=4$.

Next, we investigate the asymptotic behavior of the charges at the tips for $X \in\{4,6,8\}$. About $10^{5}$ boundary elements were used for the discretization of $\Gamma_{2}$ for each $X$. In Table 5 , the extremal values of these computations are given. The fast decrease of the minimum observed at the tip, indicates already the divergence of the charges at the tips for $X \rightarrow \infty$, as predicted in [20].

The plots of the solution on the sphere $\Gamma_{1}$ for $X \in\{4,6,8\}$ show no visible difference. We only can observe a slight increase of the maximal values, see Table 5 . On the disc at 


\begin{tabular}{l|r|r|r}
$\mathrm{a}$ & $X=4$ & $X=6$ & $X=8$ \\
\hline $\min$ & -16.9 & -212.72 & -853.54 \\
$\max$ & 1.5436 & 1.5530 & 1.5589
\end{tabular}

Table 5: Data range of the charges for $X \in\{4,6,8\}$.

$x_{1}=0$, which is opposite to the sphere, the solutions do not change for $X \in\{4,6,8\}$. We plotted the solution for $X=6$ in Fig. 3 to indicate that the charge distributions have the correct signs.
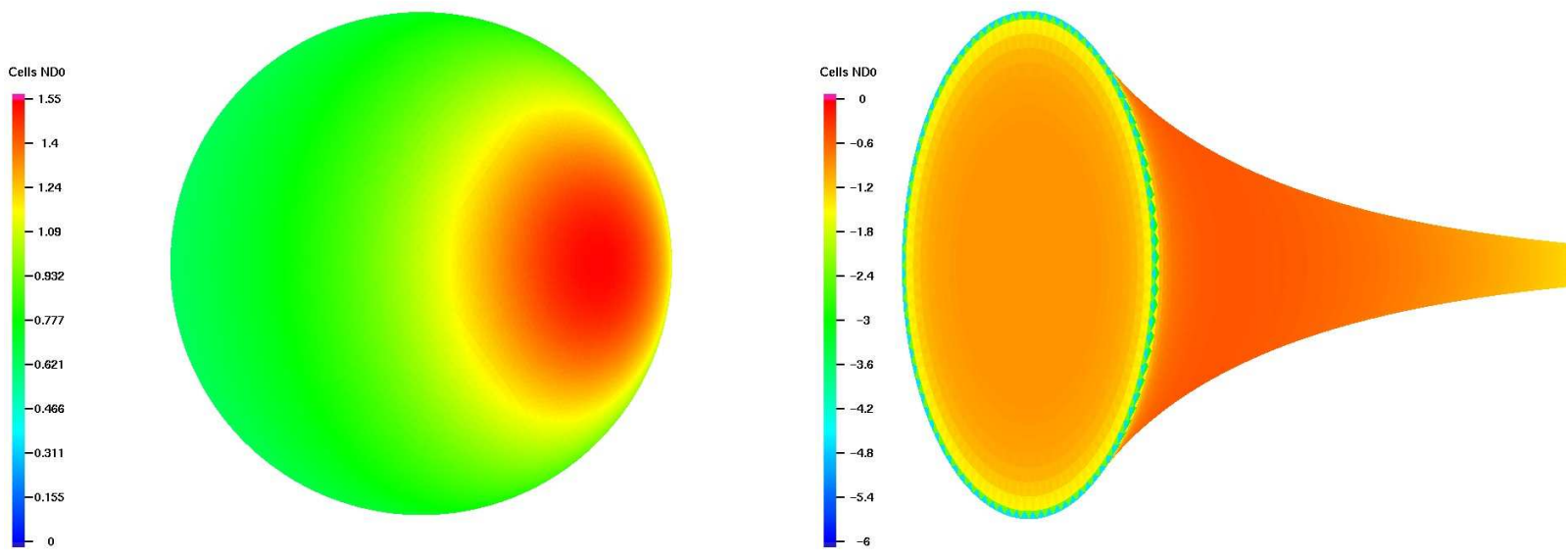

Figure 3: Charge density at the ball and the rotational body for $X=6$

Since the radius $R$ in (6.6) decreases exponentially, we had to adapt the meshsize of the boundary elements also exponentially along the body. Otherwise, it was not possible to run the simulations for $X=6$ and $X=8$. For larger $X$, the number of shape regular elements increases so rapidly that the computations were not possible anymore.

As in our first example, we are interested in the charges (6.5) at the tips for several cutoffs $X$. These values are divided by the areas of the tips, see Table 6 for $X \in\{4,6,8\}$. The computed densities at the tips indicate their divergence for $X \rightarrow \infty$ as predicted in [20].

\begin{tabular}{l|r|r|r} 
& $X=4$ & $X=6$ & $X=8$ \\
\hline $\int_{x_{1}>a} \lambda_{h} d s_{x}$ & $-1.104028 \mathrm{e}-02$ & $-1.387830 \mathrm{e}-03$ & $-1.719754 \mathrm{e}-04$ \\
$\int_{x_{1}>a} 1 d s_{x}$ & $8.715729 \mathrm{e}-04$ & $1.908049 \mathrm{e}-05$ & $3.495920 \mathrm{e}-07$ \\
density & $-12,6$ & $-72,7$ & -492
\end{tabular}

Table 6: Charges at the tips and densities for $X \in\{4,6,8\}$ and $a=X-10^{-8}$. 


\subsection{Convergence for $h \rightarrow 0$}

For the convergence behavior for $h \rightarrow 0$ and $X$ fixed in Lemma 6.1, the regularity of $\Gamma$ and, hence, the value if $s$ for $\lambda, \dot{\lambda} \in H^{s}(\Gamma)$ is needed. In both examples $\Gamma_{1} \in C^{\infty}$ whereas $\Gamma_{2}$ has an edge $\gamma=\{x=(0, \cos \varphi, \sin \varphi)\}, \varphi \in[0,2 \pi)$, where the tangent vectors on $\Gamma_{2}$ perpendicular to $\gamma$ perform a jump of angle $\frac{7}{8} \pi$ (with respect to $\Omega_{\infty}$ ). Moreover, our example is rotationally symmetric. Therefore $\lambda$ and $\dot{\lambda}$, the normal derivatives of $U_{\lambda}$ and $U_{\dot{\lambda}}$, respectively, have the form $r^{-4 / 7} \chi(r)$ where $r$ is the distance from $\gamma$ to $y=$ $\left(x_{1}, R\left(x_{1}\right) \cos \varphi, R\left(x_{1}\right) \sin \varphi\right) \in \Gamma_{2}$ for $x_{1}>0$, and to $x=(0,(1-r) \cos \varphi,(1-r) \sin \varphi) \in$ $\Gamma_{2}$ for $x_{1}=0$, and where $\chi$ is a smooth function of $r$. At the second edge of $\Gamma_{2}$ at $x_{1}=X$, the corresponding tangent vector's jump is smaller and the solutions are less singular. Using Fourier transform in $\mathbb{R}^{2}$, it then follows that $\lambda, \dot{\lambda} \in H^{s}(\Gamma)$ for $s<\frac{1}{14}$. Consequently, we find from Lemma 6.1 that

$$
\left\|\dot{\lambda}_{h}-\dot{\lambda}\right\|_{H^{t}(\Gamma)}+\left\|\lambda_{h}-\lambda\right\|_{H^{t}(\Gamma)} \leq c h^{1 / 14-t} \text { for }-\frac{15}{14} \leq t \leq s<\frac{1}{14}
$$

and

$$
\left|C_{h}-C\right|+\left|(\operatorname{cap} \mathcal{A})_{\mathrm{h}}-\operatorname{cap} \mathcal{A}\right| \leq c h^{s^{\prime}} \text { with } s^{\prime}<\frac{8}{7}
$$

\section{A Appendix}

Proof of Theorem 3.5: With Lemma 3.4 we find

$$
\mathbb{V}_{f, \varrho}\left(\varphi_{\varrho}\right)=\mathbb{V}_{f, \varrho}(\varphi)+\frac{\varrho}{2} \delta^{2} \leq \mathbb{G}_{f}(\mathcal{A}, a, g),
$$

hence,

$$
\frac{\varrho}{2} \delta^{2} \leq \mathbb{G}_{f}(\mathcal{A}, a, g)+2\left(f, \varphi_{\varrho}\right) \leq\left\{\mathbb{G}_{f}(\mathcal{A}, a, g)+2\|f\|_{H^{\frac{1}{2}(\Gamma)}} C\right\},
$$

and (3.23) follows.

For $\widetilde{\varphi}_{\varrho}^{j}=\alpha_{j} \cdot \varphi_{\varrho}^{j}$ with $\int_{\Gamma_{j}} \widetilde{\varphi}_{\varrho}^{j} g d s=a_{j}>0$, we have $\widetilde{\varphi}_{\varrho} \in \mathcal{K}(\mathcal{A}, a, g)$. Consequently,

$$
\mathbb{G}_{f}(\mathcal{A}, a, g) \leq \mathbb{V}_{f, \varrho}\left(\widetilde{\varphi}_{\varrho}\right)
$$

With $\psi:=\widetilde{\varphi}_{\varrho}-\varphi_{0}$ we have

$$
\varphi_{0}+t \psi=(1-t) \varphi_{0}+t \widetilde{\varphi}_{\varrho} \in \mathcal{K}(\mathcal{A}, a, g)
$$

for all $t \in[0,1]$ and, therefore,

$$
\left.\frac{d}{d t} F(t)\right|_{t=0} \geq 0 \text { where } F(t):=\mathbb{V}_{f, \varrho}\left(\varphi_{0}+t \psi\right)-\mathbb{V}_{f, \varrho}\left(\varphi_{0}\right)
$$


since at $t=0$ the minimum is attained. For the second derivative we find

$$
\left(\frac{d}{d t}\right)^{2} F(t)=2\|\psi\|_{V}^{2}
$$

Then with the Taylor formula about $t=0$ for the quadratic function $F(t)$ at $t=1$ and with (A.1) and (3.18), we obtain the inequality

$$
\begin{aligned}
\frac{1}{2} \cdot 2\|\psi\|_{V}^{2} \leq F(0) & +\frac{d F}{d t}(0)+\frac{1}{2} F^{\prime \prime}(0)=F(1)=\left\|\widetilde{\varphi}_{\varrho}\right\|_{V}^{2}-\left\|\varphi_{0}\right\|_{V}^{2}-2\left(f, \widetilde{\varphi}_{\varrho}-\varphi_{0}\right) \\
\|\psi\|_{V}^{2} \leq & \sum_{j=1}^{2}\left\{\left(\alpha_{j}^{2}-1\right)\left(V \widetilde{\varphi}_{\varrho}^{j}, \widetilde{\varphi}_{\varrho}^{j}\right)-\left(\alpha_{j}-1\right) 2\left(f, \varphi_{\varrho}^{j}\right)\right\} \\
& +\left(\alpha_{1} \alpha_{2}-1\right)\left(\left(V \varphi_{\varrho}^{1}, \varphi_{\varrho}^{2}\right)+\left(V \varphi_{\varrho}^{2}, \varphi_{\varrho}^{1}\right)\right) \\
& +\mathbb{V}_{f, \varrho}\left(\varphi_{\varrho}\right)-\frac{\varrho^{2}}{2} \sum_{j=1}^{2} \delta_{j}^{2}-\mathbb{G}_{f}(\mathcal{A}, a, g) ; \\
\leq & \sum_{j=1}^{2}\left|\alpha_{j}-1\right|\left\{\left(\left|\alpha_{j}-1\right|+2\right)\left(V \varphi_{\varrho}^{j}, \varphi_{\varrho}^{j}\right)+2\left|\left(f, \varphi_{\varrho}^{j}\right)\right|\right\} \\
& +\left|\alpha_{1} \alpha_{2}-1\right|\left|\left(V \varphi_{\varrho}^{1}, \varphi_{\varrho}^{2}\right)+\left(V \varphi_{\varrho}^{2}, \varphi_{\varrho}^{1}\right)\right| .
\end{aligned}
$$

With $\widetilde{\varphi}_{\varrho}^{j}=\alpha_{j} \varphi_{\varrho}^{j}$ and $a_{j}=\alpha_{j} \int_{\Gamma_{j}} \varphi_{\varrho}^{j} d s$ we have

$$
-\delta_{j}=a_{j}-\int_{\Gamma_{j}} \varphi_{\varrho}^{j} g d s=\left(\alpha_{j}-1\right) \int_{\Gamma_{j}} \varphi_{\varrho}^{j} g d s, \int_{\Gamma_{j}} \varphi_{\varrho}^{j} g d s=a_{j}+\delta_{j} \geq a_{j}-\left|\delta_{j}\right| .
$$

Hence,

$$
\begin{aligned}
\|\psi\|_{V}^{2} \leq & \sum_{j=1}^{2} \frac{\left|\delta_{j}\right|}{a_{j}-\left|\delta_{j}\right|}\left\{\left(\frac{\left|\delta_{j}\right|}{a_{j}-\left|\delta_{j}\right|}+2\right)\left\|\varphi_{\varrho}^{j}\right\|_{H^{-\frac{1}{2}(\Gamma)}}^{2}\|V\|+2 c_{D}\|f\|_{H^{\frac{1}{2}(\Gamma)}}\left\|\varphi_{\varrho}^{j}\right\|_{H^{-\frac{1}{2}(\Gamma)}}\right\} \\
& +2 \frac{\left|\delta_{1}\right|\left(a_{2}+\left|\delta_{2}\right|\right)+\left|\delta_{2}\right|\left(a_{1}+\left|\delta_{1}\right|\right)}{\left(a_{1}-\left|\delta_{1}\right|\right)\left(a_{2}-\left|\delta_{2}\right|\right)}\|V\|\left\|\varphi_{\varrho}^{1}\right\|_{H^{-\frac{1}{2}(\Gamma)}}\left\|\varphi_{\varrho}^{2}\right\|_{H^{-\frac{1}{2}(\Gamma)}} .
\end{aligned}
$$

With $a_{0}=\min \left\{a_{j}\right\}>0$ we now require $\delta \leq \frac{1}{2} a_{0}$, hence, $\varrho \geq\left(\frac{2 C_{1}}{a_{0}}\right)^{2}$, and get

$$
\|\psi\|_{V}^{2} \leq \frac{\sqrt{2}}{a_{0}}\left((3+16) C^{2}\|V\|+2 c_{D} C\|f\|_{H^{\frac{1}{2}(\Gamma)}}\right) \delta=C_{2} \delta
$$

where $C_{2}$ is given by this relation. With (3.8) and (3.23) we obtain

$$
\|\psi\|_{H^{-\frac{1}{2}(\Gamma)}}^{2} \leq C_{2} C_{1} \varrho^{-\frac{1}{2}}
$$


the desired estimate (3.24) .

The inclusion (3.25) follows from (3.16) since $\|\psi\|_{V}^{2} \geq 0$.

Acknowledgements: The second author acknowledges the Alexander von Humboldt Foundation and the National Taras Shevchenko University of Kiev for support of his visit in Kiev and the Technical University Graz for his visit in Graz. The third author acknowledges the support of the University Stuttgart for her visit in Stuttgart.

\section{References}

[1] R. Adams: Sobolev Spaces. Academic Press New York 1972.

[2] N. Bourbaki: Intégration, Chap. I-IV, Actualités Sci. Ind., 1175, Paris (1952).

[3] M. Costabel: Boundary integral operators on Lipschitz domains: Elementary results. SIAM J. Math. Anal. 19 (1988) 613-626.

[4] M. Coatabel: Some historical remarks on the positivity of boundary integral operators. In: Boundary Element Analysis (M. Schanz, O. Steinbach eds.) Springer Berlin 2007, pp.1-27.

[5] C.F. Gauss: Allgemeine Lehrsätze in Beziehung auf die im verkehrten Verhältnisse des Quadrats der Entfernung wirkenden Anziehungs- und Abstoßungs-Kräfte. (1839), Werke 5 (1867) 197-244.

[6] R. Glowinski: Numerical Methods for Nonlinear Variational Methods. Springer-Verlag Berlin 1984 (Reprint 2008).

[7] D.P. Hardin and E.B. Saff: Discretizing manifolds via minimum energy points. Notices of the AMS 51 (2004) 1186-1194.

[8] G.C. Hsiao and W.L. Wendland: The Aubin-Nitsche Lemma for integral equations. J. Integral Equations 3 (1981) 299-315.

[9] G.C. Hsiao and W.L. Wendland: Boundary Integral Equations. Springer-Verlag Berlin 2008.

[10] S.O. Kasap: Principles of Electrical Engineering Materials and Devices. Erwin McGraw Hill. Boston 1997.

[11] N.S. Landkof: Foundations of Modern Potential Theory. Springer-Verlag Berlin 1972.

[12] D. Medkova: The integral equation method and the Neumann problem for the Poisson equation on NTA domains. Integr. Eqn. Oper. Theory 63 (2009( 227-247.

[13] G. Of, O. Steinbach and W.L. Wendland: The fast multipole method for the symmetric boundary integral formulation. IMA J. Numer. Anal. 26 (2006) 272-296. 
[14] M. Ohtsuka: On potentials in locally compact spaces. J. Sci. Hiroshima Univ. Ser. A1 25 (1961) 135-352.

[15] E.B. Saff and V. Totik: Logarithmic Potentials with External Fields. Springer-Verlag Berlin 1997.

[16] O. Steinbach: Artificial multilevel boundary element preconditioners. Proc. Appl. Math. Mech. 3 (2003) 539-542.

[17] O. Steinbach: Numerical Approximation Methods for Elliptic Boundary Value Problems. Springer Science + Business, New York 2008.

[18] O. Steinbach and W.L. Wendland: On C. Neumann's method for second order elliptic systems in domains with nonsmooth boundaries. J. Math. Anal. Appl. 262 (2001) $733-748$.

[19] G. Verchota: Layer potentials and regularity for the Dirichlet problem for Laplace's eqution in Lipschitz domains. J. Functional Analysis 59 (1984) 572-611.

[20] N. Zorii: Minimal energy problem for space condensers, Ukrain. Math. Zh. 38 (1986) 431-437 (in Russian); English transl. in: Ukrain. Math. J. 38 (1986).

[21] N. Zorii: On the solvability of the Gauss variational problem. Comp. Methods Function Theory 2 (2002) 427-448.

[22] N. Zorii: Equilibrium potentials with external fields, Ukrain. Math. Zh. 55 (2003) 1178-1195 (in Russian); English transl. in: Ukrain. Math. J. 55 (2003).

[23] N. Zorii: Necessary and sufficient conditions for the solvability of the Gauss variational problem, Ukrain. Math. Zh. 57 (2005) 60-83 (in Russian); English transl. in: Ukrain. Math. J. 57 (2005).

[24] N. Zorii: On capacities of condensers in locally compact spaces. Bulletin de la Sociétée des Sciences et des Lettres de Lodz. Ser. Rech. sur les Déformations, Vol. LVI (2006) $125-142$.

[25] N. Zorii: Extremal problems to the Gauss variational problem. Ukrainian Math. Journal 58 (2006) 842-861.

[26] N. Zorii: Interior capacities of condensers with infinitely many plates in locally compact space. arxiv. org: 0906.4522v1 [math. CA] 2009. 


\section{Erschienene Preprints ab Nummer 2007/1}

2007/1 M. Windisch: Modifizierte Randintegralgleichungen für elektromagnetische Streuprobleme.

2007/2 M. Kaltenbacher, G. Of, O. Steinbach: Fast Multipole Boundary Element Method for Electrostatic Field Computations.

$2007 / 3$ G. Of, A. Schwaigkofler, O. Steinbach: Boundary integral equation methods for inverse problems in electrical engineering.

2007/4 S. Engleder, O. Steinbach: Stabilized Boundary Element Methods for Exterior Helmholtz Problems.

2007/5 O. Steinbach, G. Unger: A Boundary Element Method for the Dirichlet Eigenvalue Problem of the Laplace Operator.

2007/6 O. Steinbach, M. Windisch: Modified combined field integral equations for electromagnetic scattering.

2007/7 S. Gemmrich, N. Nigam, O. Steinbach: Boundary Integral Equations for the Laplace-Beltrami Operator.

2007/8 G. Of: An efficient algebraic multigrid preconditioner for a fast multipole boundary element method.

2007/9 O. Steinbach (ed.): Jahresbericht 2006/2007.

2007/10 U. Langer, O. Steinbach, W. L. Wendland (eds.): 5th Workshop on Fast Boundary Element Methods in Industrial Applications, Book of Abstracts

2008/1 P. Urthaler: Schnelle Auswertung von Volumenpotentialen in der Randelementmethode.

2008/2 O. Steinbach (ed.): Workshop on Numerical Simulation of the Maxwell Equations. Book of Abstracts.

2008/3 G. Of, O. Steinbach, P. Urthaler: Fast Evaluation of Newton Potentials in the Boundary Element Method.

2008/4 U. Langer, O. Steinbach, W. L. Wendland (eds.): 6th Workshop on Fast Boundary Element Methods in Industrial Applications, Book of Abstracts.

2008/5 D. Brunner, G. Of, M. Junge, O. Steinbach, L. Gaul: A Fast BE-FE Coupling Scheme for Partly Immersed Bodies

2009/1 G. Of, T. X. Phan, O. Steinbach: An energy space finite element approach for elliptic Dirichlet boundary control problems.

2009/2 G. Of, T. X. Phan, O. Steinbach: Boundary element methods for Dirichlet boundary control problems.

2009/3 G. Of, O. Steinbach: The All-Floating Boundary Element Tearing and Interconnecting Method.

2009/4 O. Steinbach: A note on the stable coupling of finite and boundary elements.

2009/5 O. Steinbach, M. Windisch: Stable boundary element domain decomposition methods for the Helmholtz equation. 